\title{
Localized theory of adsorbate-induced surface stress: Application to the Li/Mo(110) system
}

\author{
J. E. Müller, K. Dahmen, and H. Ibach \\ Institut für Schicht- und Grenzflächen Forschungszentrum Jülich, D-52425 Jülich, Germany
}

(Received 6 June 2002; published 13 December 2002)

\begin{abstract}
We present an experimental and theoretical study of surface stress in the $\mathrm{Li} / \mathrm{Mo}(110)$ system, which exhibits anisotropic behavior and nonlinear coverage dependence. At low coverages the induced stress is tensile and larger in the [ $\overline{1} 10]$ direction than in [001]. With increasing coverage $(\theta>0.25)$ both stress components switch over and become compressive. With the aid of electronic structure calculations we conclude that the anisotropy is due to the atomic arrangement characteristic of the bcc (110) surface and that the nonlinear coverage dependence is due to the combined effect of adsorbate-substrate interactions and nonlinear screening in the surface.

DOI: 10.1103/PhysRevB.66.235407

PACS number(s): 68.35.Gy, 68.35.Md, 68.35.Ja, 68.43.Fg
\end{abstract}

\section{INTRODUCTION}

The prevailing concept used in discussing surface stress induced by deposition of thin films is the lattice mismatch between film and substrate. ${ }^{1}$ However, for adsorption systems in the low-coverage limit lattice mismatch cannot be defined and a more appropriate concept is desirable to describe adsorbate induced surface stress. In this work we propose that a description of stress changes in terms of interatomic bonds becoming stronger or weaker could provide such a concept. We present an experimental and theoretical study of the $\mathrm{Li} / \mathrm{Mo}(110)$ surface which we treat as a reference for systems in which the surface bond is accompanied by a charge transfer from the adsorbate to the surface. We discuss the role of chemisorptive charge transfer, of direct adsorbate-substrate interactions, and of metal screening on the surface stress, and show that these effects lead to a description of adsorbate induced stress for low and medium coverages $(\theta<1)$ in terms of bond strengths. The $\mathrm{Li} / \mathrm{Mo}(110)$ surface represents an ideal system for the present study insofar that both the clean and the Li-covered surfaces exhibit no reconstruction. The adsorbate layer exhibits a variety of structures as a function of coverage, which are described in detail by Kanash et $a l^{2}{ }^{2}$ However, these structures exhibit an isotropic adatom distribution and do not change the symmetry of the surface.

We measured the surface stress induced by the adsorption of $\mathrm{Li}$ on the $\mathrm{Mo}(110)$ surface as a function of Li coverage, and found that (i) for low coverages the adsorbate induced stress is tensile and anisotropic with a dominant component in the [ 110$]$ direction, and (ii) for larger coverages both components of the induced stress switch over to compressive. In order to understand these results at an electronic structure level we performed total-energy calculations using the cluster method. This approach enabled us to calculate charge changes in particular bonds and in specific atoms induced by the surface bond, and lead to an explanation for the sign, magnitude, anisotropy, and nonlinear coverage dependence of the adsorbate induced surface stress in terms of molecular orbitals and atomic populations. We found that (i) the anisotropy of the surface stress is due to the atomic arrangement characteristic of the bcc (110) surface; (ii) the low coverage induced stress is determined by the charge transfer involved in the surface bond, as well as by direct interactions between the adsorbate and the surface atoms; and (iii) the nonlinear dependence on coverage is the result of the combined effect of the quenching of the direct Li-Mo interactions and of the increased metal screening that becomes nonlinear with increasing coverage. By contrast, the adsorbate-adsorbate interactions were found to play only a minor role.

\section{EXPERIMENT}

All measurements were performed under UHV conditions at pressures under $2 \times 10^{-11}$ mbar. Before each deposition the sample was cleaned by oxygen exposure. The oxygen was subsequently removed by heating the sample up to 2400 K. Surface order and cleanness was characterized with lowenergy electron diffraction (LEED) and Auger spectroscopy. Lithium was deposited on the crystal surface from a standard metal dispenser ${ }^{3}$ with a constant rate. The coverage was monitored with a Kelvin probe, via work function change. The data were calibrated using the work of Kanash et al. ${ }^{2}$ as a reference.

The adsorbate-induced surface stress was measured by the cantilever bending method (Fig. 1). In this method the change in surface stress induced by an adsorbate deposited on the one side of an elongated sample leads to the bending of the sample, which is converted into a change of the capacitance between an electrode attached to the specimen holder and a pointer welded to the sample. The displacement $\Delta d$ of the lower end of pointer was determined by

$$
\Delta d=-\epsilon_{0} A \frac{\Delta C}{C_{0}^{2}}
$$

where $C_{0}$ and $\Delta C$ are the capacity and capacity change between the pointer and the electrode, $A$ is the area of the electrode, and $\epsilon_{0}$ is the vacuum dielectric constant. The change in the curvature of the crystal was evaluated using

$$
\kappa=\frac{\Delta d}{\frac{L^{2}}{2}+L l},
$$




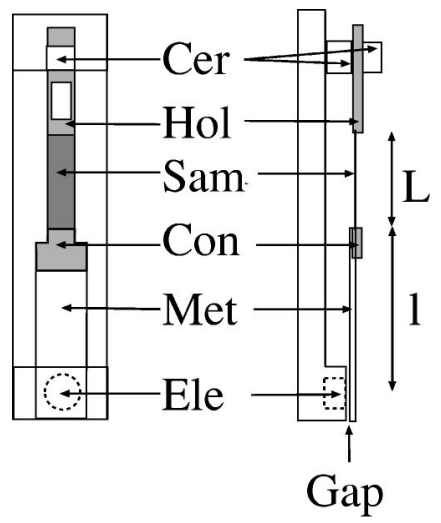

FIG. 1. Experimental arrangement showing the ceramic insulators (Cer), the specimen holder ( $\mathrm{Hol})$, the Mo(110) sample (Sam), the connector between sample and metal pointer (Con), the 1 $\times 10^{-2}$-mm-thick metal pointer (Met), the electrode (Ele), as well as the $\sim 2 \times 10^{-1}$-mm gap between metal pointer and electrode (Gap). Also indicated is the length of the sample (L) and of the pointer (1).

where $L$ is the length of the sample and $l$ the length of the pointer, as indicated in Fig. 1. We employed two different samples to study the surface stress in each of the two principal crystal orientations ([110] and [001]) of the bcc (110) surface, which we have indexed as 1 and 2, respectively. The change in surface stress $\tau^{(s)}$ can be related to the curvature of the crystal $\kappa$ by

$$
\begin{aligned}
& \kappa_{1}=\frac{6}{t_{1}^{2}}\left(s_{11}^{\prime} X_{11} \tau_{1}^{(s)}+s_{12}^{\prime} X_{12} \tau_{2}^{(s)}\right), \\
& \kappa_{2}=\frac{6}{t_{2}^{2}}\left(s_{12}^{\prime} X_{21} \tau_{1}^{(s)}+s_{22}^{\prime} X_{22} \tau_{2}^{(s)}\right),
\end{aligned}
$$

where the $s_{i j}^{\prime}$ are the transformed elastic compliances related to the bcc (110) surface, which were calculated as described elsewhere, ${ }^{4-8}$ and $X_{i j}$ are the deviations to free bending crystals due to the reduced freedom at the top and bottom ends of the samples. The values of these corrective terms are given by $X_{11}=0.994, X_{12}=0.885, X_{21}=0.803$, and $X_{22}=0.990$, and were calculated using the method of finite elements as described in a previous publication. ${ }^{4}$ The values of $\tau_{1}^{(s)}$ and $\tau_{2}^{(s)}$ were obtained by inverting Eqs. (3) and (4).

Figure 2 shows our experimental data for the adsorbate induced stress $\tau^{(s)}$ on the [110] and [001] directions of the $\mathrm{Mo}(110)$ surface as a function of Li coverage $\theta$. We employ the usual notation and denote tensile stress as positive $(\tau>0)$ and compressive stress as negative $(\tau<0)$. In the lowcoverage limit both components of the stress are tensile, the stress in the [110] direction being the larger one. The surface stress peaks at a coverage of $\theta \sim 0.25$, and eventually becomes compressive as function of $\theta$, saturating for $\theta \sim 1$. In the range $0.25<\theta<1$ the functional forms $\tau^{(s)}(\theta)$ of the two stress components are quite similar to each other, indicating that the anisotropy of the induced stress is mainly a lowcoverage effect.

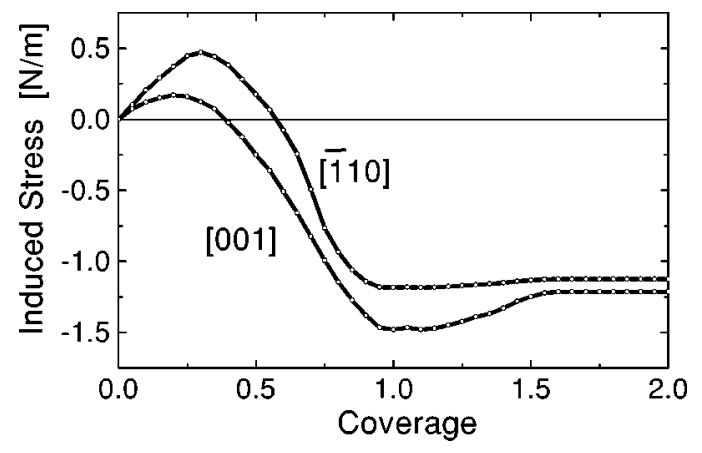

FIG. 2. Coverage dependence of the lithium induced surface stress on the $\operatorname{Mo}(110)$ surface along the $[\overline{110}]$ and [001] directions.

\section{THEORY}

We investigated the nature of the interaction between the $\mathrm{Li}$ atom and the Mo(110) surface and the physical origin of the adsorbate-induced surface stress with the aid of electronic structure calculations using the cluster approach. A small fraction of the Mo(110) surface was modeled using a $\mathrm{Mo}_{23}$ cluster, which is shown in Fig. 3, with a $\mathrm{Li}$ atom adsorbed above a long-bridge site at the center of the cluster. The one-electron problem was solved by expanding the single-particle wave functions in a basis of localized muffintin orbital (MTO) basis, ${ }^{9}$ and the exchange and correlation contributions were evaluated using the local-density approximation (LDA). For the present system the LDA overestimates binding energies and vibration frequencies by about $10 \%$, a level of accuracy that permits us to make meaningful comparison with experiment. The calculations of stress are less accurate due to the limitations of the cluster approach and have only indicative value. In spite of the limitations in numerical accuracy, our approach is well suited for the present application because the use of a localized basis provides a qualitative understanding of the numerical results in terms of well known chemical concepts, which would be difficult to achieve by other methods.

\section{A. Low-coverage limit}

We modeled the adsorption of lithium on the Mo(110) in the low-coverage limit $(\theta \rightarrow 0)$ employing the $\mathrm{Mo}_{23} \mathrm{Li}$ cluster shown in Fig. 3, and calculated the potential-energy surface of the system with the adsorbate constrained to the symmetry plane perpendicular to the surface containing the [110] direction. We found an equilibrium geometry with the $\mathrm{Li}$ atom adsorbed at a distance $z_{a}=2.22 \AA$ above the long-bridge site,

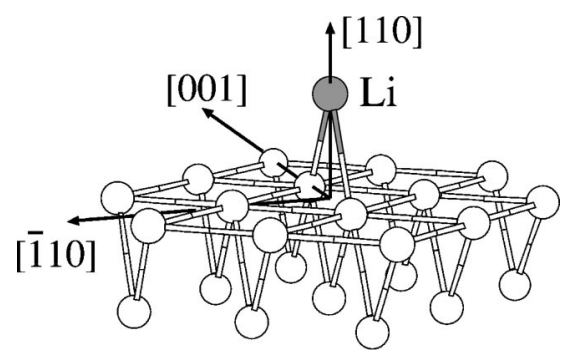

FIG. 3. $\mathrm{Mo}_{23} \mathrm{Li}$ cluster: the lines joining the atoms are only schematic and indicate the nearest-neighbor bonds of the atoms in the surface plane. 
TABLE I. Coverage dependence of the calculated binding energy $\left(E_{a}\right)$, adsorption height $\left(z_{a}\right)$, surface dipole per Li atom $(\mu)$, effective charge per $\mathrm{Li}$ atom $\left(q^{*}\right)$, vibration frequency $(\omega)$, and Li-induced surface stress $\left(\tau^{(s)}\right)$ in the $[\overline{110}]$ and [001] directions.

\begin{tabular}{lccc}
\hline \hline & $\theta \rightarrow 0$ & $\theta=0.33$ & $\theta=1$ \\
\hline$E_{a}$ & $2.64 \mathrm{eV}$ & $2.50 \mathrm{eV}$ & $2.30 \mathrm{eV}$ \\
$z_{a}$ & $2.22 \AA$ & $2.18 \AA$ & $2.13 \AA$ \\
$\omega$ & $340 \mathrm{~cm}^{-1}$ & $339 \mathrm{~cm}^{-1}$ & $329 \mathrm{~cm}^{-1}$ \\
$\mu$ & $0.82 e \AA$ & $0.76 e \AA$ & $0.44 e \AA$ \\
$q^{*}$ & $0.61 e$ & $0.57 e$ & $0.28 e$ \\
$\tau^{(s)}[\overline{110]}$ & $2.6 \mathrm{~N} / \mathrm{m} \mathrm{ML}$ & $0.8 \mathrm{~N} / \mathrm{m}$ & $-1.4 \mathrm{~N} / \mathrm{m}$ \\
$\tau^{(s)}[001]$ & $1.2 \mathrm{~N} / \mathrm{m} \mathrm{ML}$ & $0.4 \mathrm{~N} / \mathrm{m}$ & $-2.5 \mathrm{~N} / \mathrm{m}$ \\
\hline \hline
\end{tabular}

an adsorption energy $E_{a}=2.6 \mathrm{eV}$, and an induced surface dipole $\mu=0.82 e \AA$. The calculated potential energy surface was found to be extremely flat, except in regions close to the on-top sites, where it increases by up to $0.3 \mathrm{eV}$. The difference between the adsorption energies at the bridge and the threefold hollow sites amounts to about $20 \mathrm{meV}$. From the variation of the potential energy in the direction perpendicular to the surface we calculated $\mathrm{Li}$ vibration frequencies of 340,375 , and $390 \mathrm{~cm}^{-1}$, for the long-bridge, on-top, and hollow adsorption sites, respectively, which are to be compared with the low-coverage experimental value of $312 \mathrm{~cm}^{-1}$. $^{10}$ The fact that our lowest vibration frequency corresponds to adsorption at the long-bridge site confirms our assignment of that site for the low-coverage $\mathrm{Li} / \mathrm{Mo}(110)$ equilibrium geometry and is consistent with a LDA overestimation of $10 \%$ for the Li-Mo vibration. We also calculated the effective charges in the $\mathrm{Li}$ atom associated to the normal vibrations, given by $q^{*}=(1 / 2) d \mu / d z$, where the factor $1 / 2$ is due to the image charge, and $\mu$ is the surface dipole moment, and we found the same value $q^{*}=0.61 e$ for all three adsorption sites. From this result we deduce the position of the image plane to be located at $z_{i}=z_{a}-\mu / 2 q^{*}=1.53 \AA$ above the surface plane, which we define as the plane containing the Mo nuclei of the first atomic layer of the surface. Our main theoretical results for the low-coverage limit are summarized in the first column of Table I.

The surface induced stress was obtained by stretching the cluster, comparing its total energy with that of a reference nonstretched cluster, and normalizing it to unit coverage by the expression

$$
\frac{\tau^{(s)}}{\theta}=\frac{2}{S \varepsilon}\left[\Delta E_{\varepsilon}\left(\mathrm{Mo}_{23} \mathrm{Li}\right)-\Delta E_{\varepsilon}\left(\mathrm{Mo}_{23}\right)\right] .
$$

Here $\Delta E_{\varepsilon}$ is the change of the total energy when the cluster is stretched by an amount $\varepsilon, S=7.02 \AA^{2}$, is the area of the surface unit cell, and the form factor 2 is a correction for the fact that the cluster has a rhombic instead of a rectangular form. The stretching of the cluster was performed separately for the [110] and [001] directions by multiplying the linear dimensions of the cluster in each of these directions by $1+\varepsilon$ with $\varepsilon= \pm 0.01$. We obtained a tensile stress per unit coverage of $2.6 \mathrm{~N} / \mathrm{m}$ along the $[\overline{1} 10]$ direction and of $1.2 \mathrm{~N} / \mathrm{m}$ along the [001] direction, in qualitative agreement with the corresponding experimental values of $2.2 \mathrm{~N} / \mathrm{m}$ and $1.4 \mathrm{~N} / \mathrm{m}$ for the initial slope of the stress curves of Fig. 2.

In most low-coverage adsorption systems studied so far the sign and magnitude of the adsorbate induced surface stress was found to correlate with the size and magnitude of the charge transfer produced by the surface bond, ${ }^{11}$ whereby tensile stress was associated with a charge transfer from the adsorbate to the surface, and compressive stress to a transfer in the opposite direction. This correlation provides a simple model for the origin of surface stress in terms of charging or depleting the bonds in the surface. For the Li/Mo(110) system, the calculated effective charge and surface dipole reveal the existence of a charge transfer from the adsorbate to the surface which, according the charge-transfer model, would be consistent with the strengthening of the metal-metal bonds observed in the low-coverage experimental data. Our theoretical work leads us to conclude that the model is only partially correct. Inspection of the cluster wave functions shows that the charge-transfer effect is due to the interaction of the Li $2 s$ state with Mo $5 s$ states. On the clean surface, the Mo $5 s$ states lie above the Fermi level, but due to the interaction with the $\mathrm{Li} 2 s$ state, the Mo $5 s$ states move to lower energies and become locally occupied leading to increased Mo-Mo bonding and giving rise to a tensile stress contribution, in agreement with the charge-transfer model. ${ }^{11}$ However, the interaction between the $\mathrm{Li} 2 s$ and the Mo $5 s$ states also gives rise to a direct adsorbate-metal bonding, which in the low-coverage limit is the dominant tensile contribution to the surface stress. In this context it is important to point out that the Mo $4 d$ states do not interact with the Li $2 s$ state. The Li $2 s$ state is so extended $(\sim 8 \AA)$ in space that it is essentially constant over the volume of the $5 d$ states, such that the positive and negative parts of the $5 d$ functions cancel out in the overlap integral. It is also worth pointing out that the bonding states involved in the charge transfer are not necessarily located close to the Fermi level. This means that discussions of charge transfer based on density of states arguments alone ${ }^{12}$ are misleading and may even predict the wrong sign for the induced surface stress.

\section{Bonding and atomic charges}

In order to characterize the local electronic structure in the vicinity of the adsorption site, we define pair charges between the $i$ th and $j$ th atoms of the cluster, given by ${ }^{13}$

$$
Q(i, j)=2 \sum_{n}^{\text {occ }} \sum_{\lambda, \lambda^{\prime}}\left\langle\Psi_{n} \mid \chi_{i, \lambda}\right\rangle\left\langle\chi_{j, \lambda^{\prime}} \mid \Psi_{n}\right\rangle,
$$

where $\chi_{i, \lambda}$ are MTO basis functions centered on the $i$ th atom with angular momentum $\lambda=(l, m)$, and the $n$ summation extends over all occupied states $\Psi_{n}$ of the cluster. The pair charges are an implementation of the standard Mulliken populations, ${ }^{14}$ and are independent of the basis set provided that the latter is reasonably chosen. The off-diagonal term, $Q(i, j)$ with $i \neq j$, can be positive or negative and may be interpreted, respectively, as the bonding or antibonding charge between the $i$ th and $j$ th atoms. The diagonal term $Q(i, i)$, which is positive definite, represents the atomic 
TABLE II. Redistribution of the Li $2 s$ electron charge induced by the adsorption of the $\mathrm{Li}$ atom on the $\mathrm{Mo}_{23}$ cluster of Fig. 3, as deduced from calculated bonding charges and projected in the [110], [001], and vertical directions.

\begin{tabular}{lcccc}
\hline \hline & Total & {$[\overline{1} 10]$} & {$[001]$} & Vertical \\
\hline Li atom & $Q_{1}=0.08$ & & & \\
Li-Mo bonds & $Q_{2}=0.54$ & 0.11 & 0.16 & 0.27 \\
Mo-Mo bonds & $Q_{3}=0.19$ & 0.20 & 0.04 & -0.05 \\
Mo atoms & $Q_{4}=0.18$ & & & \\
\hline \hline
\end{tabular}

charge on the $i$ th atom not involved in the bonding to other atoms, and provides the screening of its core charge $Z(i)$. In the following we shall loosely interpret $Q(i, j)$ as proportional to the orbital interaction between the $i$ th and $j$ th atoms, separated by a distance $d_{i, j}$, and $[Q(i, i)-Z(i)]$ $\times[Q(j, j)-Z(j)] / d_{i, j}$ as the corresponding descreening repulsion.

In order to study the effect of the Li-Mo interaction on the electronic structure of the surface and of the adsorbate, we will be interested in the change of the bonding charges induced by the surface bond. Accordingly, we define paircharge changes, $\Delta Q(i, j)$, by subtracting from Eq. (6) the corresponding values of the bare $\mathrm{Mo}_{23}$ cluster without the adsorbate. In this representation, the electron charge on the adsorbate is given by $Q(\mathrm{Li}, \mathrm{Li})+1 / 2 \sum_{j \in \mathrm{Mo}_{23}} \Delta Q(\mathrm{Li}, j)$ $=0.35 e$, to be compared with the value of $1-q^{*}$ $=0.39 e$, deduced above from the calculated effective charge. As a result of the surface bond the charge of the Li $2 s$ electron splits up in four partial charges, which we listed in Table II as follows: $Q_{1}=Q(\mathrm{Li}, \mathrm{Li})=0.08 e$ remain localized on the adatom providing a limited screening of the Li nucleus; $Q_{2}=\sum_{j \in \mathrm{Mo}_{23}} Q(\mathrm{Li}, j)=0.54 e$ are transferred to $\mathrm{Li}-\mathrm{Mo}$ bonds, where it gives rise to a direct attractive interaction between the adsorbate and the neighboring Mo surface atoms; $Q_{3}=\sum_{i, j, \in \mathrm{Mo}_{23}}^{i \neq j} \Delta Q(i, j)=0.19 e$ are transferred to Mo-Mo bonds in the metal surface, where it produces a strengthening of these bonds; and $Q_{4}=\sum_{i \in \mathrm{Mo}_{23}} \Delta Q(i, i)$ $=0.18 e$ are transferred to the metal atoms, where it increases the screening of the atoms. The last three terms yield tensile contributions to the surface stress, the $Q_{4}$ term being the less important. Although the $Q_{3}$ and $Q_{4}$ contributions are of the same order of magnitude, the tensile effect due to the bonding charges $Q_{3}$ outweighs that of the atomic charges. This effect is due to the fact that the bonding charges are localized in the region between two atoms and provide an optimal screening of the nuclear charges. The atomic charges are isotropically distributed around the nuclear charges and are much less efficient in screening the same charges. This point is discussed below in detail.

The main features discussed above can be visualized in Fig. 4, which shows the redistribution of charge induced by the surface bond, as given by the charge density difference, $\Delta \rho=\rho\left(\mathrm{Mo}_{23} \mathrm{Li}\right)-\rho\left(\mathrm{Mo}_{23}\right)-\rho(\mathrm{Li})$, in the symmetry plane perpendicular to the surface containing the [110] direction. The continuous and dashed lines represent increase and decrease of charge, and point to the regions of increased and

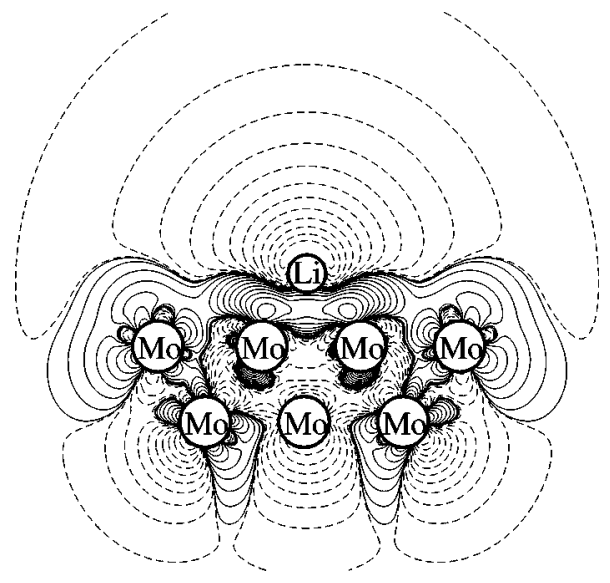

FIG. 4. Charge densities difference $\Delta \rho$ induced by the adsorption of $\mathrm{Li}$ at the long-bridge site of a $\mathrm{Mo}_{23}$ cluster. The continuous $(\Delta \rho>0)$ and dashed $(\Delta \rho<0)$ contour lines represent densities given by $2 n^{3} \times 10^{-4} e \AA^{-3}$, for $n=-15, \ldots, 15$.

decreased bonding. Note the large charge depletion on the volume of the long-ranged $\mathrm{Li} 2 s$ state, which is transferred to the surface! This charge piles up preferentially in the region between the adatom and the surface, giving rise to the bonding $(\Delta \rho>0)$ chemical interaction between the Li atom and the metal, and corresponds to the $Q_{2}$ term discussed above. Note also that the charge density in the space between the Mo atoms actually decreases, which appears to be in contradiction with the charge transfer to Mo-Mo bonds reported above. This charge decrease is due to the occupation of Mo $4 d$ antibonding states as a result of the local decrease of the work function of the metal induced by the adsorbate. However, this effect is more than compensated by the charge transfer to the extended Mo $5 s$ states interacting with the Li $2 s$ state in the region where the large charge accumulation takes place. This region corresponds roughly to the position of the image plane.

\section{Anisotropy of the surface stress}

We projected the bonding charge changes onto the [110], [001], and vertical directions by multiplying the bonding charge changes $\Delta Q(i, j)$ by the squared cosines of the directions of the corresponding $i-j$ bonds, and calculated the integrated values of these projected charges over the entire cluster (Table II). The integrated projected charges show that the dominant part of the induced surface stress anisotropy is due to the Mo-Mo bonds, which exhibit a [110] component which is much larger than the [001] one. This effect reflects the topological anisotropy of the bcc (110) surface. Note in Fig. 3 that the displacements of the surface atoms in the [001] and [110] directions are weakly coupled, and that the interplanar metal bonds between the first and second metal layers are exclusively contained in the vertical plane containing the [001] direction. When the bonds in the surface plane expand or contract, the atoms on the surface plane are free to move along the [110] direction while their motion in the [001] direction is restricted by the interplanar bonds. This means that the stress along the $[\overline{1} 10]$ direction originates mainly in changes in the bonds contained in the surface 

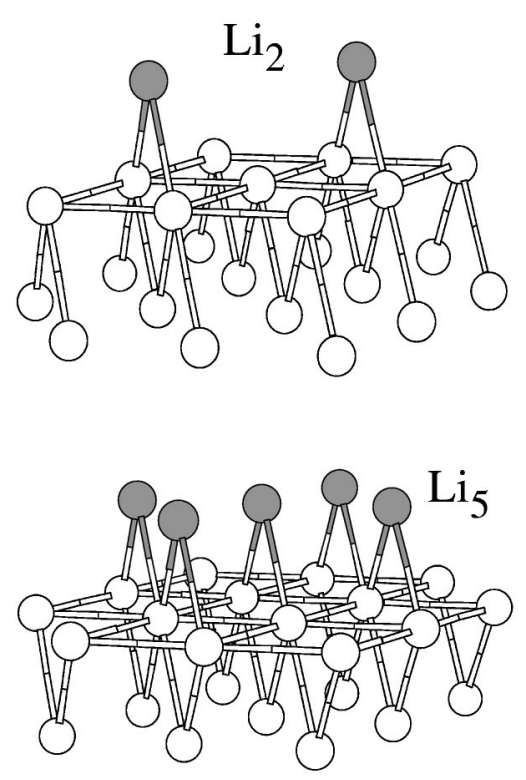

FIG. 5. Clusters (a) $\mathrm{Mo}_{23} \mathrm{Li}_{2}$ and (b) $\mathrm{Mo}_{23} \mathrm{Li}_{5}$, modeling Li coverages of $\theta=0.33$ and $\theta=1$ on the Mo(110) surface. The lines joining the atoms are only schematic and indicate the nearest-neighbor bonds of the atoms in the surface plane.

plane while the stress along [001] is due to changes in the vertical bonds of the interplanar interaction.

The direct Li-Mo interaction can be split into a vertical attractive component giving rise to the actual surface bond and a lateral component that contributes to the surface stress. The Li-Mo contribution to the surface stress exhibits also a small anisotropy. The lateral Li-Mo interaction is somewhat larger in the [001] than in the [110] direction, reflecting the denser distribution of atoms in that direction. This contribution to the stress anisotropy is of opposite sign than that of the Mo-Mo interactions. Note in Table II that the interplanar bonds expand and give rise to a compressive stress that cancels out the in-plane contribution, such that the [001] stress arises exclusively from the direct $\mathrm{Li}-\mathrm{Mo}$ interactions and the Mo-atomic charges. The latter are isotropic and do not contribute to the anisotropy of the stress.

\section{B. Finite-coverage regime}

We modeled the coverage dependence of the Li-induced stress with the $\mathrm{Mo}_{23} \mathrm{Li}_{2}$ and $\mathrm{Mo}_{23} \mathrm{Li}_{5}$ clusters simulating the local geometries of the $c(1 \times 3)$ and $(1 \times 1)$ structures, which correspond to coverages of 0.33 and 1.0 , respectively. In the calculations presented below we assumed that all the $\mathrm{Li}$ atoms adsorb above long-bridge sites, as shown in Fig. 5. We did explore other geometries, including hollow and on-top sites, but they all showed substantially weaker bonding energies, which means that they are not likely to be found under normal experimental conditions. For $\theta=0.33$ we found a ground-state geometry with the Li atoms adsorbed $2.14 \AA$ above the surface and a binding energy of $2.5 \mathrm{eV}$ per $\mathrm{Li}$ atom. We calculated the vibration frequency by letting one of the two $\mathrm{Li}$ atoms oscillate, and obtained the value $\omega$ $=339 \mathrm{~cm}^{-1}$. The surface dipole and effective charge per $\mathrm{Li}$ atom were given by $\mu=0.76 e \AA$ and $q^{*}=0.57 e$. The sur- face stress was calculated using Eq. (5) with the appropriate $\theta$ value, and we found $\tau^{(s)}[110]=0.3 \mathrm{~N} / \mathrm{m}$ and $\tau^{(s)}[001]$ $=-1.9 \mathrm{~N} / \mathrm{m}$, which may be compared with experimental values of $0.2 \mathrm{~N} / \mathrm{m}$ and $-0.3 \mathrm{~N} / \mathrm{m}$, respectively. For $\theta=1.0$ the binding energy was $E_{a}=2.3 \mathrm{eV}$, the adsorption height, $z_{a}$ $=2.13 \AA$, and the vibration frequency, calculated by letting only the central $\mathrm{Li}$ atom oscillate, was given by $\omega$ $=329 \mathrm{~cm}^{-1}$. The induced surface dipole and the effective charge per $\mathrm{Li}$ atom were given by $\mu=0.44 e \AA$, and $q^{*}$ $=0.28 e$, from which we deduce the location of the image plane to be located $1.34 \AA$ above the surface. The calculated values for the surface stress were $\tau^{(s)}[\overline{1} 10]=-1.4 \mathrm{~N} / \mathrm{m}$ and $\tau^{(s)}[001]=-2.4 \mathrm{~N} / \mathrm{m}$, which are consistent with the measured experimental values of $-1.2 \mathrm{~N} / \mathrm{m}$ and $-1.5 \mathrm{~N} / \mathrm{m}$, respectively. These calculated values have been included in Table I.

\section{Vibration frequencies and work-function change}

The calculated vibration frequencies and surface dipoles enable us to make contact with experiment, and to obtain a useful assessment of the numerical validity of the cluster approach. The comparison with experiment also gives insight about the magnitude of the dipole-dipole interaction and of the depolarization fields, arising on the charged adsorbed layer. The surface dipole can be related to the change of the work function according to $\Delta \phi=2 \pi \theta \mu / S$, where $S$ is the area of the surface unit cell. We find $\Delta \phi$ values of 1.8 and $3.0 \mathrm{eV}$ for coverages of 0.33 and 1.0, which may be compared with the experimental values of 2.4 and $2.0 \mathrm{eV}$, respectively. ${ }^{10}$ The numerical discrepancy at smaller coverages mirrors the lateral and vertical smallness of the cluster, both of them leading to a decreased value for the surface dipole: (i) the reduced depth of the cluster means that the image charge is not completely formed and shifted towards the surface; and (ii) the reduced lateral extension of the cluster means that our calculation does not include all the Li-Mo bonds which would occur in an infinite surface. The numerical discrepancy at $\theta=1$ reflects the fact that the cluster calculation does not include the long-ranged depolarization field due to the charged adlayer, that tends to reduce the surface dipole. Note that the errors due to cluster size and missing depolarization tend to cancel each other, such that the error at high coverages is larger than the direct comparison seem to indicate.

Our calculated vibration frequencies exhibit a slight weakening of the surface bond with increasing coverage that mirrors the corresponding variation of the binding energy. In order to compare with experiment we corrected our calculated frequencies by the effect of the dipole-dipole interaction. Using the effective charges calculated above and assuming an electronic polarizability $\alpha_{e}=4 \AA^{3}$ for the adsorbed species, we obtain ${ }^{11}$ corrected vibration frequencies for coverages 0.33 and 1.0 that are 24 and $14 \mathrm{~cm}^{-1}$ above the $\theta \rightarrow 0$ value, respectively. Kröger et al. ${ }^{10}$ found a frequency increase of $\Delta \omega=30 \mathrm{~cm}^{-1}$ for $\theta=0.30$ and a small decrease $\Delta \omega=-5 \mathrm{~cm}^{-1}$ for $\theta=1$. The source of error for the calculated frequencies are the same as for the work function changes: (i) at low coverages our effective charge $q^{*}$ is probably too small as a result of the limited lateral extension of the cluster, and (ii) at high coverages is probably too large 
TABLE III. Redistribution of the Li $2 s$ electron charge induced by $\mathrm{Li}$ adsorption on the $\mathrm{Mo}_{23}$ cluster with a coverage $\theta=1$, as shown in Fig. 5(b), as deduced from calculated bonding charges and projected in the [110], [001], and vertical directions.

\begin{tabular}{lllll}
\hline \hline & Total & {$[\overline{1} 10]$} & {$[001]$} & Vertical \\
\hline Li-Li bonds & 0.09 & & & \\
Li atom & 0.16 & & & \\
Li-Mo bonds & 0.54 & & & \\
Mo-Mo bonds & 0.12 & 0.14 & 0.02 & -0.03 \\
Mo atoms & 0.24 & & & \\
\hline \hline
\end{tabular}

because of the missing depolarization field in our calculation. In assessing these discrepancies we must also keep in mind that our results are based on the assumption that the Li atoms adsorb at long-bridge sites, while the experiments of Kröger et $a l .{ }^{10}$ were done for intermediate coverages, where lack of registry between adsorbate and substrate may be assumed. As pointed out above, the potential energy surface for $\mathrm{Li}$ adsorption is quite flat, which means that the occupation of sites intermediate between the long bridge and the hollow is likely. As was pointed out above adsorption sites other than the long-bridge sites exhibit a larger frequency.

\section{Nonlinear coverage dependence}

Table I summarizes our theoretical results as a function of coverage. Observe that while the increasing coverage brings about only weak changes in the configurational properties (bonding geometry, binding energy, vibrational frequency), the induced surface stress and the quantities characterizing the Li-Mo charge transfer (static dipole moment and effective charge), show a strong coverage dependence. This qualitative correlation between surface stress and charge transfer is compounded by the fact that both the stress and the work function become independent of coverage for $\theta>1,{ }^{10}$ and suggests the existence of some relation between them. Note, however, that while the charge per Li atom, as given by $q^{*}$, decreases with increasing coverage, the amount of charge transferred per unit area increases from $0.61 e$ for $\theta \rightarrow 0$, to $2 \times 0.57=1.14 e$ for $\theta=0.33$, and to $5 \times 0.28=1.4 e$ for $\theta=1$, which indicates that the explanation of the compressive character of the induced stress must involve effects not present in the low-coverage limit. These new effects are (i) the Li-Li interaction; (ii) the quenching of the Li-Mo interaction; and (iii) the nonlinear screening of the Mo atomic charges.

We evaluated the Li-Li interaction by stretching the adsorbed $\mathrm{Li}_{2}$ and $\mathrm{Li}_{5}$ systems keeping constant the lattice parameter of the underlying $\mathrm{Mo}_{23}$ cluster and found that it gives rise to very small compressive stress contributions of $0.05 \mathrm{~N} / \mathrm{m}$ and $0.2 \mathrm{~N} / \mathrm{m}$ for $\theta=0.33$ and $\theta=1$, respectively. The reason for the smallness of the $\mathrm{Li}-\mathrm{Li}$ interaction is the accumulation of screening charge between the Li atoms with increasing coverage, displayed in Table I by the corresponding decrease of $q^{*}$. More detailed insight is provided by the pair charges changes induced by the surface bond, which we summarized in Table III for the $\mathrm{Li}_{5} / \mathrm{Mo}_{23}(\theta=1)$ system.
Note that the increasing repulsion between $\mathrm{Li}$ ions is balanced by an increase in the Li atomic charges and by the development of Li-Li bond charges with increasing coverage, leading to a negligible net contribution of the $\mathrm{Li}-\mathrm{Li}$ interaction to the coverage dependence of the induced surface stress. This result is consistent with the conclusions of a previous study of the $\mathrm{CO} / \mathrm{Ni}(100)$ system. ${ }^{15}$

The second effect that occurs at finite coverages is the progressive quenching of the adsorbate-substrate interaction. Observe in Table III that the Li-Mo bonding charges remain quite large compared with their low-coverage value, mirroring the weak coverage dependence of the binding energy. However, as the coverage increases each surface Mo atom becomes attracted to more and more Li atoms uniformly distributed on the surface, such that the net component of the force in the surface plane tends to cancel out, and so does the contribution of the Li-Mo interaction to the induced surface stress. The quenching of the Li-Mo interaction switches on when the distance between adsorbates becomes comparable with the range of the $\mathrm{Li} 2 s$ state, which is about $8 \AA$. Thus we expect this quenching to begin for $\theta \sim 0.25$, which is roughly the coverage value where the function $\tau^{(s)}(\theta)$ begins to turn over to compressive values in Fig. 2. However, while this effect suppresses an appreciable source of tensile stress, it cannot yield a net compressive stress as observed in the experiment.

The compressive surface stress at finite coverages is the reaction of the metal-metal interactions to the large amount of charge transferred to the surface. While, as was pointed out above, in the low-coverage limit the charge transferred to the metal distributes evenly between bonding and atomic states, for $\theta \sim 1$ the charge is found to go preferentially to atomic states, which are predominantly antibonding. Note, with reference to Table I, that the charge per surface unit cell in the Mo-Mo bonds increases from $0.19 e$ in the lowcoverage limit to $5 \times 0.12=0.60 e$ for $\theta=1$, while the atomic Mo charges increase from $0.18 e$ to $5 \times 0.24=1.20 e$. What appears as a charge transfer from the adsorbate to metal atomic states is actually an intrametal charge transfer from bonding to antibonding states. Inspection of the cluster wave functions show that the Li $2 s$ state interacts exclusively with Mo-Mo bonding $5 s$ states and that the charge transfer to the atomic states is an indirect process. The increased charge in the bonding states move these states higher in energy, and as they reach the Fermi level charge is transferred to antibonding states. The antibonding states are less sensitive to charging effects than the bonding states because they are more delocalized and can therefore take much more charge without moving to higher energies. The intrametal charge transfer from bonding to antibonding states is a characteristic signature of screening: the charge transferred to the surface arranges itself as to reduce the extension of electric fields to a minimum, leading to the preferential occupation of delocalized states.

The fact that overscreening of the nuclei at higher coverages leads to compressive stress can be also understood as resulting from the electrostatic repulsion due to the charging of the first metal layer. This effect can be visualized with an incomplete but instructive model of two-dimensional point 


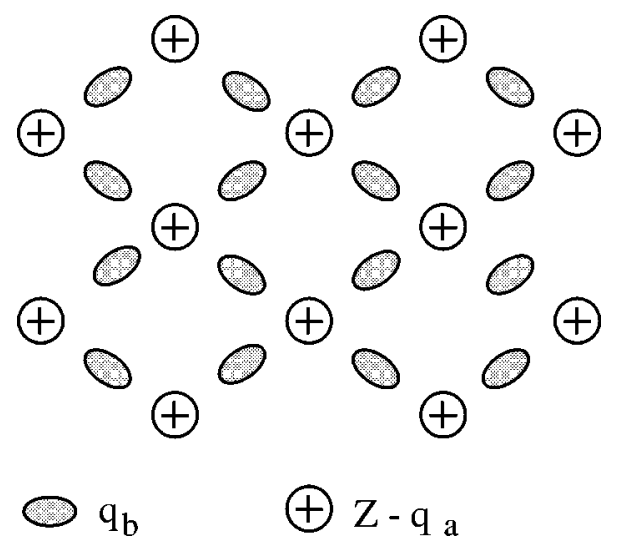

FIG. 6. Point-charge model of the Mo(110) surface plane, showing the position of the nuclei $(+)$ with its screening charges $q_{a}$ as well as the bonding charges $q_{b}$ located in the midpoint between Mo atoms.

charges illustrated schematically in Fig. 6. This model involves screening charges $Z-q_{a}$ located at Mo lattice positions and bonding charges $q_{b}$ at the middle between two lattice positions, and leads to a force in the layer given by

$$
F=f_{1}(d) q_{b}\left(Z-q_{a}\right)-f_{2}(d)\left(Z-q_{a}\right)^{2}-f_{3}(d) q_{b}^{2},
$$

where $f_{1}, f_{2}$, and $f_{3}$ are two-dimensional Madelung constants that depend on the lattice parameter $d$, and $Z=6$ is the effective Mo nuclear charge seen by the valence electrons. The $f_{1}$ term describes the interaction between the bonding charges and the partially screened nuclei, the $f_{2}$ term the screened nuclei-nuclei repulsion, and $f_{3}$ the repulsion between bonding charges. The first term is the dominant one for it involves the smaller distances between interacting charges and is responsible for the change of sign of the induced surface stress with increasing coverage. At low cover- ages the transferred charge goes to $q_{b}$ and the $f_{1}$ term is tensile. With increasing coverage the transferred charge goes mainly to $q_{a}$, which amounts to a decrease of the effective nuclear charge leading to a compressive induced stress.

The nonlinear screening is essentially an isotropic effect, and do not contribute to the anisotropy of the induced stress. As we pointed out in Sec. III A 2, the stress anisotropy arises in the flow of charge to bonding states, a process which becomes suppressed for $\theta>0.25$, leading to a similar functional form for the coverage dependence in both the [110] and the [001] directions.

\section{Summary and outlook}

We presented a localized theory of surface stress which we applied to the $\mathrm{Li} / \mathrm{Mo}(110)$ system, and showed that it describes all the features that appear in the experimental data, which exhibits anisotropic behavior and nonlinear coverage dependence. Our theory is based in the consideration of bond strengths and atomic screening, which we characterize in terms of bonding charges derived from standard population analysis. We showed that the $\mathrm{Li}$ atom interacts exclusively with bonding states of the underlying metal, leading to a charge transfer to these states, which is responsible for the tensile stress observed at for low coverages. With increasing coverage the bonding states become flooded with charge and move up to higher energies, triggering a secondary charge transfer from bonding to antibonding states, which gives rise to a compressive surface stress. The compressive behavior at higher coverages appears to occur for adsorbates that bind with transfers of charge to or from the surface which are so large, that they give rise to nonlinear metal screening. We have found that the nonlinear screening response of the metal occurs not only for alkali metals but also for electronegative adsorption systems line oxygen and $\mathrm{CO}$ (on hollow sites) where the charge transfer opposite the opposite direction. ${ }^{16}$
${ }^{1}$ J.H. van der Merwe, D.L. Tönsing, and P.M. Stoop, Surf. Sci. 312, 387 (1994); J.H. van der Merwe, J. Woltersdorf, and W.A. Jesser, Mater. Sci. Eng. 81, 1 (1986); I. V. Markov, Crystal Growth for Beginners (World Scientific, Signapore, 1995), Chap. 4.

${ }^{2}$ O.V. Kanash and A.G. Fedorus, Zh. Eksp. Teor. Fiz. 86, 223 (1984) [Sov. Phys. JETP 59, 1 (1984)].

${ }^{3}$ SEAS Getters S. P. A., Milan.

${ }^{4}$ K. Dahmen, S. Lehwald, and H. Ibach, Surf. Sci. 446, 161 (1999).

${ }^{5}$ D. Sander, Rep. Prog. Phys. 62, 809 (1999).

${ }^{6}$ J. F. Nye, Physical Properties of Crystals (Clarendon, Oxford, 1972).

\footnotetext{
${ }^{7}$ W.A. Brantley, J. Appl. Phys. 44, 534 (1973).

${ }^{8}$ L.H. He and C.W. Lim, Surf. Sci. 478, 203 (2001).

${ }^{9}$ J.E. Müller, R.O. Jones, and J. Harris, J. Chem. Phys. 79, 1874 (1983).

${ }^{10}$ J. Kröger, D. Bruchmann, S. Lehwald, and H. Ibach, Surf. Sci. 449, 227 (2000).

${ }^{11}$ H. Ibach, Surf. Sci. Rep. 29, 193 (1997).

${ }^{12}$ P.J. Feibelman, Phys. Rev. B 56, 2175 (1997).

${ }^{13}$ J.E. Müller, Surf. Sci. 178, 589 (1986).

${ }^{14}$ R.S. Mulliken, J. Chem. Phys. 23, 1833 (1955).

${ }^{15}$ H. Ibach, Electrochim. Acta 45, 575 (1999).

${ }^{16}$ J. E. Müller and H. Ibach (unpublished).
} 